\title{
A Prospective Study of Risk Factors for Bacterial Vaginosis in HIV-1-Seronegative African Women
}

\author{
R. Scott McClelland, MD, MPH ${ }^{1,2,4}$, Barbra A. Richardson, $\mathrm{PhD}^{3}$, Susan M. Graham, MD, \\ $\mathrm{MPH}^{1,4}$, Linnet N. Masese, MBChB ${ }^{2,4}$, Ruth Gitau, MBChB ${ }^{4}$, Ludo Lavreys, MD, PhD $^{2,4}$, \\ Kishorchandra Mandaliya, MBChB ${ }^{5}$, Walter Jaoko, MBChB, MMed, $\mathrm{PhD}^{4}$, Jared M. Baeten, \\ MD, PhD ${ }^{1}$, and Jeckoniah O. Ndinya-Achola, MBChB, MMed, MSc ${ }^{4}$ \\ ${ }^{1}$ Department of Medicine, University of Washington, Seattle, USA \\ ${ }^{2}$ Department of Epidemiology, University of Washington, Seattle, USA \\ ${ }^{3}$ Department of Biostatistics, University of Washington, Seattle, USA \\ ${ }^{4}$ Department of Medical Microbiology, University of Nairobi, Nairobi, Kenya \\ ${ }^{5}$ Coast Provincial General Hospital, Mombasa, Kenya
}

\begin{abstract}
Background-Bacterial vaginosis (BV) is common and has been associated with increased HIV-1 susceptibility. The objective of this study was to identify risk factors for BV in African women at high risk for acquiring HIV-1.
\end{abstract}

Methods-We conducted a prospective study among 151 HIV-1-seronegative Kenyan female sex workers. Non-pregnant women were eligible if they did not have symptoms of abnormal vaginal itching or discharge at the time of enrollment. At monthly follow-up, a vaginal examination and laboratory testing for genital tract infections were performed. Multivariate Andersen-Gill proportional hazards analysis was used to identify correlates of BV.

Results-Participants completed a median of 378 (interquartile range 350-412) days of followup. Compared to women reporting no vaginal washing, those who reported vaginal washing 1-14 (adjusted hazard ratio [aHR] 1.29, 95\% confidence interval [CI] 0.88-1.89), 15-28 (aHR 1.60, 95\% CI 0.98-2.61), and >28 times/week (aHR 2.39, 95\% CI 1.35-4.23) were at increased risk of $\mathrm{BV}$. Higher BV incidence was also associated with the use of cloth for intravaginal cleansing (aHR 1.48, 95\% CI 1.06-2.08) and with recent unprotected intercourse (aHR 1.75, 95\% CI 1.472.08). Women using depot medroxyprogesterone acetate contraception were at lower risk for BV (aHR $0.59,95 \%$ CI $0.48-0.73$ ).

Conclusions-Vaginal washing and unprotected intercourse were associated with increased risk of BV. These findings could help to inform the development of novel vaginal health approaches for HIV-1 risk reduction in women.

\section{Keywords}

Bacterial vaginosis; vaginal washing; intravaginal practices; women; Africa

Corresponding Author: R. Scott McClelland, MD, MPH, University of Washington, Box 359909, $3259^{\text {th }}$ Avenue, Seattle, WA 98104, Fax: (206) 543-4818, Telephone: (206) 543-4278, mcclell@u.washington.edu. 


\section{INTRODUCTION}

Bacterial vaginosis (BV) has been associated with increased risk for HIV-1 acquisition, ${ }^{1-4}$ genital tract infections, ${ }^{5}$ pelvic inflammatory disease, ${ }^{6}$ and preterm delivery of a low-birthweight infant. ${ }^{7}$ Prospective studies have begun to shed light on the natural history of BV, and on the importance of specific demographic, behavioral, and biological risk factors for this condition. African ancestry, ${ }^{8}$ lower educational level, ${ }^{8}$ higher number of sexual partners, ${ }^{8,9}$ and oral-vaginal contact have all been associated with BV. ${ }^{10}$ In contrast, condom use and hormonal contraception may be protective. ${ }^{11,12}$ Nonetheless, important questions remain about the role of some risk factors for BV. For example, intravaginal practices are common among African women and may increase the risk of $\mathrm{BV}$, providing a potential mechanism through which intravaginal practices might increase HIV-1 susceptibility. ${ }^{13-15}$ In addition, while it is generally accepted that hydrogen-peroxide producing Lactobacillus colonization is associated with lower risk of BV, 2, 16 the extent to which other BV risk factors act through effects on vaginal lactobacilli is not known.

We recently completed a randomized trial of monthly periodic presumptive treatment as an intervention to reduce vaginal infections in Kenyan women at risk for HIV-1. ${ }^{17}$ A secondary aim was to utilize the prospectively collected data from the trial to identify modifiable risk factors for BV in this population.

\section{MATERIALS AND METHODS}

\section{Population and Procedures}

Detailed methods for the randomized trial are presented elsewhere. ${ }^{17}$ Because the orally administered study drugs reduced the incidence of BV in the trial population, the present analysis focuses on women who were randomized to receive the matching oral placebo (hard gel capsules filled with lactose monohydrate). Briefly, non-pregnant, 18-45 year old, HIV-1-seronegative sex workers were eligible to enroll. The majority of women recruited to the cohort were bar workers who supported their regular income with occasional transactional sex. The protocol for the trial required that women did not have symptoms of abnormal vaginal itching or discharge at the time of enrollment. Subsequent visits were included in this analysis regardless of whether symptoms were present.

At baseline and at monthly follow-up visits, women completed an interview covering sexual risk behavior and personal hygiene practices during the past 7 days. In order to distinguish between external vulvar versus internal vaginal washing, we used a two-part question that was pilot tested prior to the study. Women were first asked whether they cleaned inside the vagina. Those who responded 'yes' were asked to clarify how far inside the vagina they cleaned, differentiating between 'less than a finger tip length' versus 'more than a finger tip length' beyond the vaginal introitus. We defined 'finger tip length' as the length of the distal phalanx of the second finger (approximately 2 centimeters). Women were classified as performing intravaginal washing if they reported vaginal cleaning practices that extended more than a finger tip length beyond the introitus.

At each visit, women had a pelvic speculum examination with collection of specimens for laboratory diagnosis of genital tract infections. Vaginal $\mathrm{pH}$ was measured using a test strip (ColorpHast 4.0-7.0, EM Reagents), and vaginal secretions were tested for the release of an amine odor after addition of a drop of $10 \%$ potassium hydroxide. Blood was collected for HIV-1 serological testing and a urine pregnancy test was performed. Women who reported abnormal vaginal itching or discharge were treated syndromically with metronidazole 2 grams as a single dose plus clotrimazole $200 \mathrm{mg}$ vaginal suppositories nightly for three nights. Participants were invited to return for their laboratory results one week after each 
examination. If additional treatment for sexually transmitted infections was indicated on the basis of the laboratory results, medications were provided according to WHO and Kenya Ministry of Health Guidelines. ${ }^{18}$ Asymptomatic BV and vaginal candidiasis were not treated, as there is currently no indication for treatment of these conditions in non-pregnant women without symptoms. Women in the trial were asked to return for 12 monthly followup visits. Participation was discontinued if they acquired HIV-1 or became pregnant. Individual risk reduction education including instructions on condom use and a free supply of condoms were provided at each visit. Participants were counseled that vaginal washing may increase the risk for HIV-1, and were encouraged to minimize or eliminate these practices. This study was approved by the ethical review committees at Kenyatta National Hospital and the University of Washington. All participants provided written informed consent.

\section{Laboratory Methods}

Serological screening for HIV-1 was performed using an enzyme linked immunosorbent assay (ELISA; Detect-HIV; BioChem ImmunoSystems). Positive samples were confirmed using a second ELISA (Recombigen; Cambridge Biotech or Vironostika; Biomeriux). ${ }^{19}$ Urine pregnancy testing was performed using a rapid $\beta$-hCG test (Plasmatec Laboratory Products).

Gram stained slides of vaginal secretions were evaluated according to standardized microscopic scoring criteria. ${ }^{20} \mathrm{~A}$ vaginal saline wet mount was examined microscopically for the presence of budding yeast or hyphae, clue cells, and Trichomonas vaginalis. A drop of $10 \%$ potassium hydroxide was added, and the slide was examined a second time for the presence of yeast. Gram stained slides of endocervical secretions were examined microscopically. The number of polymorphonuclear leukocytes (PMN) in three nonadjacent high-power fields was counted, and the average cervical PMN count was calculated.

Lactobacillus culture was performed on Rogosa agar (Difco TM, Becton Dickinson), and production of $\mathrm{H}_{2} \mathrm{O}_{2}$ was assessed by sub-culture on tetramethylbenzadine agar with horseradish peroxidase. ${ }^{21}$ Culture for T. vaginalis was performed in Diamond's modified media and culture for Neisseria gonorrhoeae was performed on modified Thayer-Martin media.

\section{Statistical Methods}

Analyses were performed using SPSS (version 15; SPSS) and S-Plus 2000 (Mathsoft). All women randomized to the placebo arm of the trial were considered eligible for inclusion in this analysis. Univariate and multivariate analyses of risk factors for incident BV were performed using Andersen-Gill proportional hazards models with robust variance estimates. This method of modeling allows for multiple events within a single individual over time. Variables associated with BV on univariate analysis $(\mathrm{P} \unlhd 0.10$ ) were included in the multivariate models. Linear regression was used to assess the dose-response relationship between the frequency of vaginal washing and BV.

As in prior analyses, ${ }^{11}$ we assumed an effect window of 85 days to capture the effect of hormonal contraceptive use on incident BV among women who changed or discontinued contraceptive methods (70 days of persistent effect of hormonal contraception after discontinuation +15 days from $\mathrm{BV}$ acquisition to detection at a clinic visit, assuming acquisition at the midpoint between monthly visits). The window of effect for Lactobacillus on subsequent $\mathrm{BV}$ was set at 60 days, which allowed us to capture the presence of lactobacilli during $1-2$ preceding visits. 
The primary endpoint for this analysis was BV by microscopic criteria. ${ }^{20}$ In addition, we analyzed risk factors for BV diagnosed on the basis of clinical criteria, defined as the identification of at least three out of four clinical signs: abnormal vaginal discharge, $\mathrm{pH}$ $>4.5$, clue cells present on vaginal saline wet preparation, and the release of an amine odor on addition of a drop of $10 \%$ potassium hydroxide to vaginal fluid. ${ }^{22}$ Vaginal candidiasis was defined as the identification of budding yeast or hyphae on the vaginal saline wet mount or potassium hydroxide preparation. Women were considered to have T. vaginalis infection if motile trichomonads were observed on the vaginal wet mount or in culture.

\section{RESULTS}

Between May 2003 and November 2005, 378 women were screened for eligibility, of whom 310 were enrolled. Of the 68 women who did not enroll, 65 declined or did not return for a scheduled enrollment visit, one planned to leave the area, one reported intolerance to metronidazole, and one was pregnant. One hundred and fifty five women were randomized to the placebo arm, and 152 of these women returned for at least one follow-up visit. One woman was excluded from these analyses because she seroconverted to HIV-1 at her first follow-up visit, and therefore did not contribute HIV-1-seronegative follow-up time. The remaining 151 women are the focus of the longitudinal analyses presented here. The median duration of follow-up was 378 (interquartile range [IQR] 350-412) days per participant and the median interval between visits was 30 (IQR 28-35) days. Overall, the women contributed a total of 153 person-years of follow-up over 1,570 visits.

Baseline characteristics of the 151 sex workers included in these analyses are presented in Table 1. The median age of participants was 32 (IQR 27-39) years. They reported a relatively low number of sexual partners (median 1, IQR $0-1$ ), and a low frequency of intercourse (median 1, IQR 0-2) during the past week. One-hundred and thirty (86\%) women reported vaginal washing with a median frequency of 14 (14-21) times per week. The most common combination of practices, reported by 86 (57\%) women, was use of a finger to wash inside the vagina with soap or antiseptic both during bathing and after sex. Although the women denied symptoms of abnormal vaginal itching or discharge at enrollment, BV was present by Gram stain criteria in 56 (37\%) and by clinical criteria in 27 $(18 \%)$ participants.

\section{Risk Factors for Bacterial Vaginosis}

Bacterial vaginosis was identified by Gram stain criteria at 553 follow-up visits (3.61 visits with BV per woman-year). Risk factors for BV by microscopic criteria are presented in Table 2. Several modifiable risk factors were associated with BV in univariate analyses. To ascertain the independent effects of each of these risk factors, an initial multivariate model was constructed including all covariates that were associated with BV in univariate analyses (P $\unlhd .10$ ), except Lactobacillus, since changes in vaginal Lactobacillus colonization could represent an intermediate step in the causal linkage between BV and other risk factors. In this model, more frequent vaginal washing, use of cloth to clean inside the vagina, unprotected intercourse, and $T$. vaginalis infection were independently associated with increased risk of BV. In contrast, the risk of BV was significantly lower in women using depot medroxyprogesterone acetate (DMPA) contraception and in those with concurrent vaginal candidiasis. A second multivariate model was constructed including vaginal Lactobacillus status. The absence of Lactobacillus remained a significant predictor of BV. However, addition of this variable did not substantially change the hazard ratios for any of the other covariates, suggesting that vaginal washing, use of cloth inside the vagina, unprotected intercourse, DMPA, and other vaginal infections may influence the risk of BV 
through mechanisms that are at least partly independent of their effects on vaginal lactobacilli.

Vaginal washing practices may vary in relation to vaginal symptoms like odor or discharge. For this reason, we repeated the analyses in Table 2 using the vaginal washing practices reported at study enrollment, as these practices would be unrelated to subsequent symptoms. Baseline vaginal washing frequency was correlated with the average frequency of vaginal washing during follow-up $(\mathrm{R}=0.52, \mathrm{p}<0.001)$. Women who reported the highest frequency of vaginal washing at baseline remained at a significantly higher risk of BV by Gram stain criteria during subsequent follow-up. Compared to women reporting no vaginal washing at baseline, the adjusted hazard ratios for BV in those who reported at enrollment that they performed vaginal washing 1-14 times per week, 15-28 times per week, and $>28$ times per week were 1.06 (95\% confidence interval [CI] 0.83-1.35), 0.80 (95\% CI 0.63-1.02), and 1.55 (95\% CI 1.16-2.06) respectively. Similar results were observed upon further adjustment for vaginal Lactobacillus status (data not shown).

Bacterial vaginosis was identified by clinical criteria at 300 visits (1.96 visits with BV per woman-year). Because there was a lower rate of BV by clinical criteria compared to Gram stain criteria, analyses of risk factors for BV by clinical criteria had lower statistical power. In general, risk factors for BV measured by clinical criteria were similar to risk factors identified when BV was diagnosed microscopically (Table 3 ). However, the association between vaginal washing and BV by clinical criteria was not statistically significant in multivariate analysis.

\section{DISCUSSION}

This prospective study identified several modifiable risk factors associated with BV in this cohort of Kenyan sex workers. Vaginal washing and unprotected intercourse were both associated with increased risk of BV. In contrast, use of the injectable progesterone contraceptive DMPA was associated with a lower rate of BV.

Accumulating evidence from prospective studies of women in a variety of settings, and using very different intravaginal practices, suggests that women's risk for BV may be increased by common vaginal cleansing practices. Our data demonstrating an increased risk of BV in women who perform vaginal washing using fingers or cloth are consistent with a recently published study among US women, which demonstrated that vaginal douching (using a stream of liquid) increased the risk of BV in those with intermediate vaginal flora by Gram stain. ${ }^{23}$ The dose-response relationship observed in our study provides additional epidemiological evidence in favor of a causal association. We also found a significant association between BV and the use of cloth to clean inside the vagina. These results, which are similar to our findings in an earlier cross-sectional study, ${ }^{24}$ have implications for planning interventions aimed at modifying intravaginal practices. In particular, substantial reductions in $\mathrm{BV}$ might be achieved by reducing the frequency of vaginal washing or by modifying specific practices, even if complete cessation of vaginal washing proves difficult.

There is accumulating evidence that sexual risk behavior may influence the risk of $\mathrm{BV}$, but different studies have provided somewhat conflicting results about the importance of specific risk factors. For example, in a multi-center study of US women, monogamy was associated with a significantly lower risk of $\mathrm{BV} .{ }^{8}$ This finding contrasts with the results from an Australian cohort in which having a regular sexual partner was associated with increased risk, while having a new partner was associated with lower risk..$^{25}$ The present study highlights the importance of unprotected intercourse as a risk factor for BV regardless of the number or type (new versus regular) of sexual partners. This contrasts with the 
epidemiology of traditional sexually transmitted pathogens like gonorrhea and Chlamydia, for which new sex partners play a particularly important role in transmission. ${ }^{26}$

The finding that DMPA may lower the risk of BV is consistent with prior studies demonstrating similar associations. ${ }^{11,27}$ Changes in vaginal mucosal immune cell populations have been demonstrated in women using DMPA, likely reflecting altered local immune function. ${ }^{28}$ This could in turn influence the risk for BV. Injectable progesterone contraception may also modify the risk of BV by reducing menstrual frequency and volume. $^{29}$

Vaginal Lactobacillus colonization is an important mediator of the risk of vaginal infections. ${ }^{2,16}$ However, the present data suggest that the relationship between vaginal infections and specific risk factors such as intravaginal practices, sexual risk behavior, and hormonal contraceptive use, may not be explained entirely on the basis of their impact on lactobacilli. An important caveat to these results is the fact that some Lactobacillus species such as L. iners may be common among African women, ${ }^{30}$ and can be difficult to culture successfully. Studies using cultivation-independent techniques to identify and quantify vaginal bacterial populations could provide important insights into the pathogenesis and natural history of disturbances of the vaginal flora. ${ }^{31}$

We used microscopic scoring criteria for the diagnosis of BV in the primary analyses presented here. ${ }^{20}$ The risk factors for BV by clinical criteria were generally similar, ${ }^{22}$ although there were fewer episodes using this definition, resulting in lower statistical power. Microscopic criteria were selected for defining the primary BV endpoint because $86 \%$ of these women reported vaginal washing, raising concern about whether the clinical criteria would produce reliable results. Specifically, we felt that vaginal washing might influence the examiners' ability to identify abnormal vaginal discharge, which is a component of the clinical definition of BV.

The findings from this prospective study differ in a few important respects from those of our earlier cross-sectional analysis of baseline data in this patient population. ${ }^{24}$ In the crosssectional study, BV was associated with use of petroleum jelly for vaginal lubrication, use of saliva for vaginal lubrication, and less frequent bathing. These associations were attenuated (lubrication with petroleum jelly) or eliminated (lubrication with saliva, less frequent bathing) in the longitudinal analysis. There are a number of possible explanations for the difference in these results. First, the cross-sectional analysis included only asymptomatic enrollment visits, whereas the longitudinal study included follow-up visits regardless of whether symptoms were present. Second, time-varying adjustment for potential confounding factors in the longitudinal analysis may have provided greater control of confounding. Finally, the associations may have occurred by chance in the cross-sectional study (Type-1 error), or the longitudinal study may have failed to show associations that truly exist (Type-2 error). Further research in other populations is needed to address these questions.

There were limitations to this study. Accurate assessment of sexual risk can be difficult. We have previously demonstrated that self-reported sexual behaviors in this population are associated with increased risk for sexually transmitted diseases. ${ }^{32}$ Nonetheless, there remains a potential for residual confounding. It is also important to consider the fact that all participants in this study reported transactional sex. Their sexual practices, vaginal washing practices, and risk for BV may differ from women who do not engage in sex work. To date, there are limited data comparing intravaginal practices across populations. ${ }^{13}$ Finally, all participants in this study were asymptomatic at enrollment. However, women were not excluded if they had a prior history of symptomatic vaginal conditions, and were retained after enrollment regardless of whether they became symptomatic. While the recruitment of 
asymptomatic women differs from many studies of BV, we feel that this population may be particularly important, since women frequently do not recognize symptoms of BV. Indeed, the high frequency of asymptomatic BV was highlighted by the $37 \%$ baseline prevalence in our study.

The importance of understanding risk factors for BV has become increasingly evident in light of numerous studies linking this condition to HIV-1 acquisition. ${ }^{1-4}$ The results presented here identify several modifiable risk factors for $\mathrm{BV}$, which could help to inform the development of interventions to improve vaginal health. Ultimately, interventions aimed at reducing or eliminating vaginal washing could provide a simple, inexpensive, femalecontrolled strategy for reducing women's risk of acquiring HIV-1 infection.

\section{Acknowledgments}

Financial Support: National Institutes of Health (grant K23 AI52480). Fogarty International Center (grant T43TW00007 to S.M.G. and L.N.M.).

We are particularly grateful to the women who contributed their time and effort to make this study a success. We also wish to recognize the valuable contributions made by our clinic and laboratory staff and by our administrative team. We thank the Mombasa Municipal Council for allowing us to use their clinical facilities, and Coast Provincial General Hospital for providing laboratory space.

\section{References}

1. Martin HL Jr, Nyange PM, Richarson BA, et al. Hormonal contraception, sexually transmitted diseases, and risk of heterosexual transmission of human immunodeficiency virus type 1 . J Infect Dis. 1998; 178:1053-59. [PubMed: 9806034]

2. Martin HL, Richardson BA, Nyange PM, et al. Vaginal lactobacilli, microbial flora, and risk of human immunodeficiency virus type 1 and sexually transmitted disease acquisition. J Infect Dis. 1999; 180:1863-8. [PubMed: 10558942]

3. Taha TE, Hoover DR, Dallabetta GA, et al. Bacterial vaginosis and disturbances of vaginal flora: association with increased acquisition of HIV. AIDS. 1998; 12:1699-706. [PubMed: 9764791]

4. Myer L, Denny L, Telerant R, Souza M, Wright TC Jr, Kuhn L. Bacterial vaginosis and susceptibility to HIV infection in South African women: a nested case-control study. J Infect Dis. 2005; 192:1372-80. [PubMed: 16170754]

5. Wiesenfeld HC, Hillier SL, Krohn MA, Landers DV, Sweet RL. Bacterial vaginosis is a strong predictor of Neisseria gonorrhoeae and Chlamydia trachomatis infection. Clin Infect Dis. 2003; 36:663-8. [PubMed: 12594649]

6. Peipert JF, Montagno AB, Cooper AS, Sung CJ. Bacterial vaginosis as a risk factor for upper genital tract infection. Am J Obstet Gynecol. 1997; 177:1184-7. [PubMed: 9396917]

7. Hillier SL, Nugent RP, Eschenbach DA, et al. Association between bacterial vaginosis and preterm delivery of a low-birth-weight infant. The Vaginal Infections and Prematurity Study Group. N Engl J Med. 1995; 333:1737-42. [PubMed: 7491137]

8. Ness RB, Kip KE, Soper DE, Stamm CA, Rice P, Richter HE. Variability of bacterial vaginosis over 6- to 12-month intervals. Sex Transm Dis. 2006; 33:381-5. [PubMed: 16543864]

9. Barbone F, Austin H, Louv WC, Alexander WJ. A follow up study of methods of conatraception, sexual activity, and rates of trichomoniasis, candidiasis, and bacterial vaginosis. Am J Obstet Gynecol. 1990; 163:510-514. [PubMed: 2167008]

10. Schwebke JR, Richey CM, Weiss HL. Correlation of behaviors with microbiological changes in vaginal flora. J Infect Dis. 1999; 180:1632-6. [PubMed: 10515826]

11. Baeten JM, Nyange PM, Richardson BA, et al. Hormonal contraception and risk of sexually transmitted disease acquisition: results from a prospective study. Am J Obstet Gynecol. 2001; 185:380-5. [PubMed: 11518896] 
12. van De Wijgert JH, Mason PR, Gwanzura L, et al. Intravaginal practices, vaginal flora disturbances, and acquisition of sexually transmitted diseases in Zimbabwean women. J Infect Dis. 2000; 181:587-94. [PubMed: 10669342]

13. WHO. Multi-country study on gender, sexuality, and vaginal practices. 2007.

14. Myer L, Kuhn L, Stein ZA, Wright TC, Denny L. Intravaginal practices, bacterial vaginosis, and women's susceptibility to HIV infection: epidemiological evidence and biological mechanisms. Lancet Infect Dis. 2005; 5:786-794. [PubMed: 16310150]

15. McClelland RS, Lavreys L, Hassan WM, Mandaliya K, Ndinya-Achola JO, Baeten JM. Vaginal washing and increased risk of HIV-1 acquisition among African women: a 10-year prospective study. AIDS. 2006; 20:269-73. [PubMed: 16511421]

16. Hawes SE, Hillier SL, Benedetti J, et al. Hydrogen peroxide producing lactobacilli and acquisition of vaginal infections. J Infect Dis. 1996; 174:1058-1063. [PubMed: 8896509]

17. McClelland RS, Richardson BA, Hassan WM, et al. Improving Vaginal Health in Women at Risk for HIV-1: Results of a Randomized Trial. J Infect Dis. In press.

18. WHO. Guidelines for the management of sexually transmitted infections. Geneva: World Health Organization; 2003. p. 1-91.

19. WHO, CDC. Guidelines for Appropriate Evaluations of HIV Testing Technologies in Africa. Harare; Zimbabwe: 2001. p. 1-48.

20. Nugent RP, Krohn MA, Hillier SL. Reliability of diagnosing bacterial vaginosis is improved by a standardized method of gram stain interpretation. J Clin Microbiol. 1991; 29:297-301. [PubMed: 1706728]

21. Eschenbach DA, Critchlow CW, Watkins H, et al. A dose-duration study of metronidazole for the treatment of nonspecific vaginosis. Scand J Infect Dis Suppl. 1983; 40:73-80. [PubMed: 6364332]

22. Amsel R, Totten PA, Spiegel CA, Chen KCS, Eschenbach DA, Holmes KK. Nonspecific vaginitis. Diagnostic criteria and microbial and epidemiologic associations. Am J Intern Med. 1983; 74:1422.

23. Hutchinson KB, Kip KE, Ness RB. Vaginal douching and development of bacterial vaginosis among women with normal and abnormal vaginal microflora. Sex Transm Dis. 2007; 34:671-5. [PubMed: 17413534]

24. Hassan WM, Lavreys L, Chohan V, et al. Associations Between Intravaginal Practices and Bacterial Vaginosis in Kenyan Female Sex Workers Without Symptoms of Vaginal Infections. Sex Transm Dis. 2007; 34:384-388. [PubMed: 17065846]

25. Bradshaw CS, Morton AN, Hocking J, et al. High recurrence rates of bacterial vaginosis over the course of 12 months after oral metronidazole therapy and factors associated with recurrence. $\mathrm{J}$ Infect Dis. 2006; 193:1478-86. [PubMed: 16652274]

26. Marrazzo JM. A persistent(ly) enigmatic ecological mystery: bacterial vaginosis. J Infect Dis. 2006; 193:1475-7. [PubMed: 16652273]

27. Riggs M, Klebanoff M, Nansel T, Zhang J, Schwebke J, Andrews W. Longitudinal Association Between Hormonal Contraceptives and Bacterial Vaginosis in Women of Reproductive Age. Sex Transm Dis. 2007 Publish Ahead of Print.

28. Ildgruben AK, Sjoberg IM, Hammarstrom ML. Influence of hormonal contraceptives on the immune cells and thickness of human vaginal epithelium. Obstet Gynecol. 2003; 102:571-82. [PubMed: 12962945]

29. Belsey EM. Vaginal bleeding patterns among women using one natural and eight hormonal methods of contraception. Contraception. 1988; 38:181-206. [PubMed: 2971505]

30. Anukam KC, Osazuwa EO, Ahonkhai I, Reid G. Lactobacillus vaginal microbiota of women attending a reproductive health care service in Benin city, Nigeria. Sex Transm Dis. 2006; 33:5962. [PubMed: 16385223]

31. Fredricks DN, Fiedler TN, Marrazzo JM. Molecular identification of bacteria associated with bacterial vaginosis. N Engl J Med. 2005; 355:1899-911. [PubMed: 16267321]

32. McClelland RS, Lavreys L, Katingima C, et al. Contribution of HIV-1 infection to acquisition of sexually transmitted disease: a 10-year prospective study. J Infect Dis. 2005; 191:333-8. [PubMed: 15633091] 
Table 1

Baseline Characteristics of Female Sex Workers in Enrolled Mombasa, Kenya between May 2003 and November $2005(\mathrm{~N}=151)$

\begin{tabular}{|c|c|}
\hline Variable & Value \\
\hline Age, median (IQR), years & $32(27-39)$ \\
\hline Duration of sex work, median (IQR), years & $4.3(1.6-8.1)$ \\
\hline Education, median (IQR), years & $8(7-11)$ \\
\hline Bar worker ${ }^{a}$ & $116(76.8 \%)$ \\
\hline Tobacco use & $18(11.9 \%)$ \\
\hline Alcohol use & $115(76.2 \%)$ \\
\hline Vaginal washing in past week & $130(86.1 \%)$ \\
\hline Frequency, median (IQR), per week ${ }^{b}$ & $14(14-21)$ \\
\hline \multicolumn{2}{|l|}{ Vaginal washing substance $b$} \\
\hline Water only & $43(33.1 \%)$ \\
\hline Soap or antiseptic ${ }^{c}$ & $87(66.9 \%)$ \\
\hline \multicolumn{2}{|l|}{ Vaginal washing method $b$} \\
\hline Finger & $127(97.7 \%)$ \\
\hline Cloth & $3(2.3 \%)$ \\
\hline \multicolumn{2}{|l|}{ Timing of vaginal washing $d$} \\
\hline Before sex & $6(4.7 \%)$ \\
\hline After sex & $128(100 \%)$ \\
\hline During bathing & $127(99.2 \%)$ \\
\hline After urinating & $35(27.3 \%)$ \\
\hline Other & $1(<1 \%)$ \\
\hline Bathing frequency, median (IQR), per week & $14(14-14)$ \\
\hline \multicolumn{2}{|l|}{ Vaginal lubricant for sex } \\
\hline Vaseline & $10(6.7 \%)$ \\
\hline Saliva & $6(4.0 \%)$ \\
\hline Sex partners, median (IQR), in past week & $1(0-1)$ \\
\hline Sex frequency, median (IQR), in past week & $1(0-2)$ \\
\hline Any unprotected intercourse in past week & $32(21.2 \%)$ \\
\hline New sex partner in past month & $22(14.7 \%)$ \\
\hline Any history of vaginal intercourse & $152(100 \%)$ \\
\hline Any history of anal intercourse & $0(0 \%)$ \\
\hline Any history of oral-vaginal contact & $5(3.3 \%)$ \\
\hline \multicolumn{2}{|l|}{ Contraception } \\
\hline None or tubal ligation & $93(61.6 \%)$ \\
\hline $\mathrm{OCP}$ & $10(6.6 \%)$ \\
\hline DMPA & $43(28.5 \%)$ \\
\hline Norplant & $3(2.0 \%)$ \\
\hline IUD & $2(1.3 \%)$ \\
\hline
\end{tabular}




\begin{tabular}{|lc|}
\hline Variable & Value \\
\hline Bacterial vaginosis (Gram stain criteria) & $56(37.1 \%)$ \\
Bacterial vaginosis (clinical criteria) & $27(17.9 \%)$ \\
Lactobacillus (any) & $13(8.6 \%)$ \\
Lactobacillus $\left(\mathrm{H}_{2} \mathrm{O}_{2}\right.$ producing) & $3(2.0 \%)$ \\
Vaginal candidiasis & $15(9.9 \%)$ \\
Trichomonas vaginalis & $2(1.3 \%)$ \\
Neisseria gonorrhoeae & $0(0 \%)$ \\
Cervicitis &
\end{tabular}

NOTE: Data are no. (\%) of subjects unless otherwise specified. DMPA, depot medroxyprogesterone acetate; $\mathrm{H}_{2} \mathrm{O}_{2}$, hydrogen peroxide; IUD, intrauterine device; $\mathrm{OCP}$, oral contraceptive pill.

${ }^{a}$ Bar work has been associated with an increased risk of sexually transmitted infections and HIV acquisition in the Mombasa Cohort ${ }^{1}$.

${ }^{b} \mathrm{~N}=130$ women who reported vaginal washing.

${ }^{c}$ Use of antiseptic for vaginal washing was reported by 3 women.

$d_{\mathrm{N}}=128$. Data were missing for 2 of the 130 women who reported vaginal washing.

${ }^{e}$ Average polymorphonuclear leukocyte count 230 cells per high power field on microscopy of Gram stained cervical secretions. 


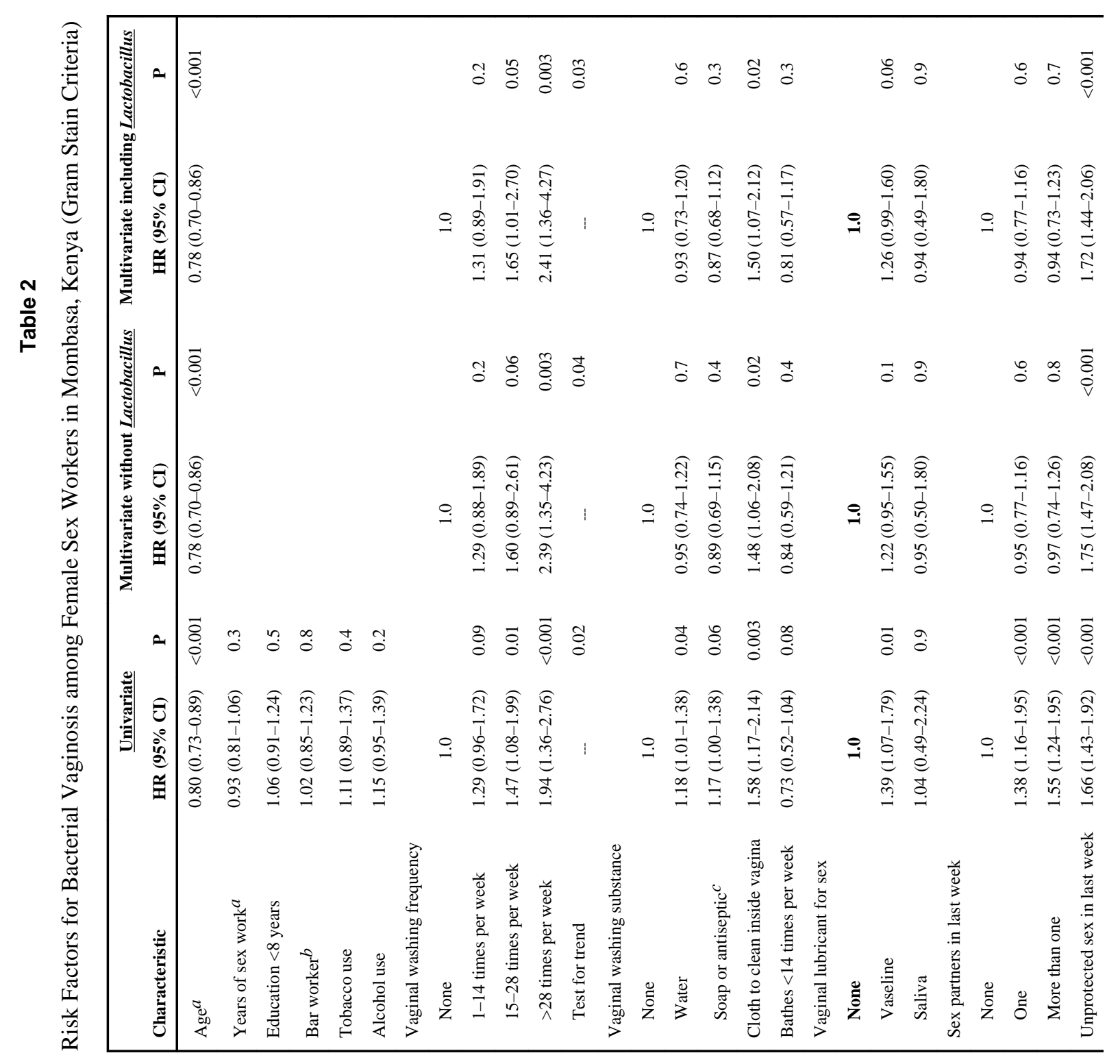




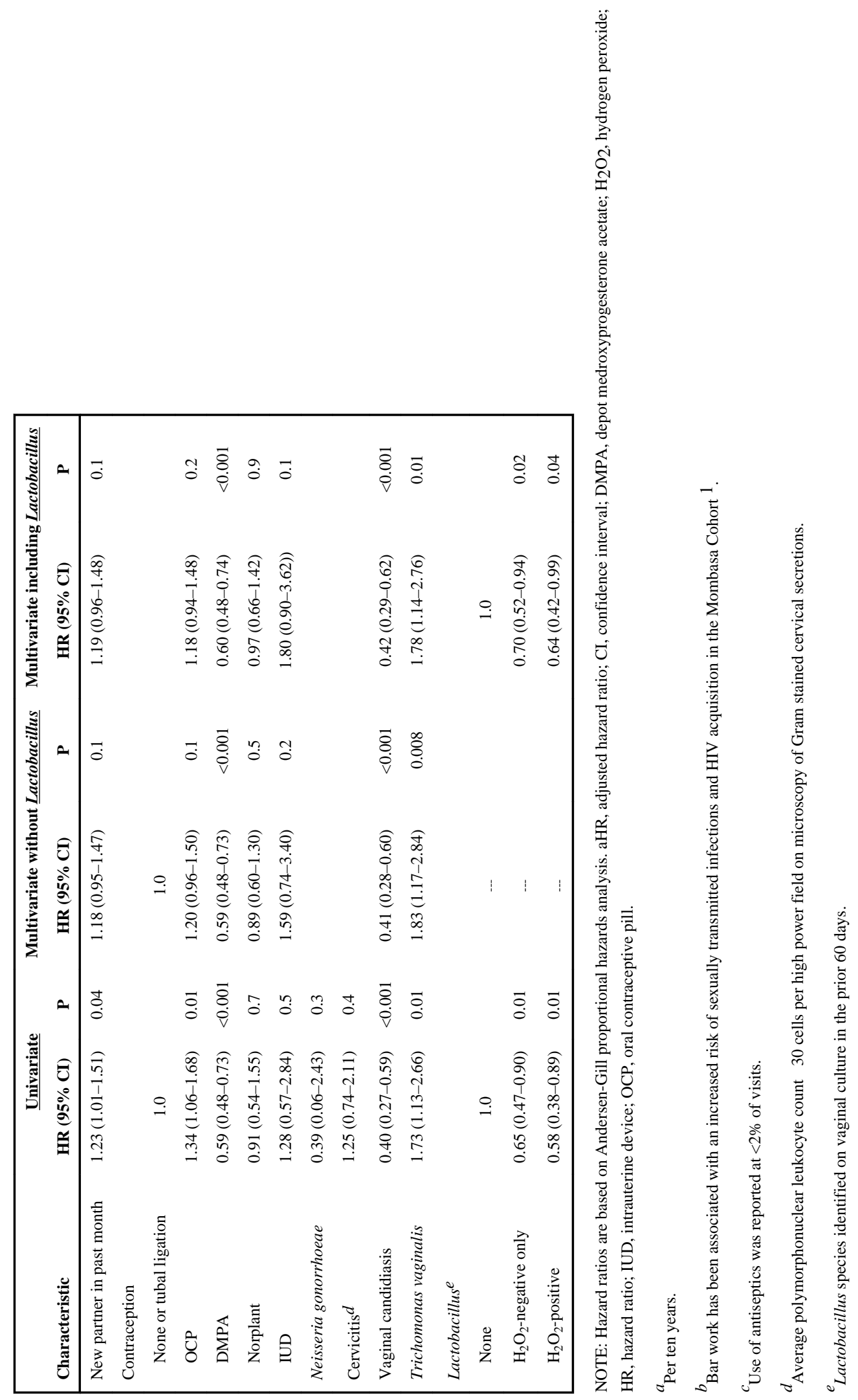




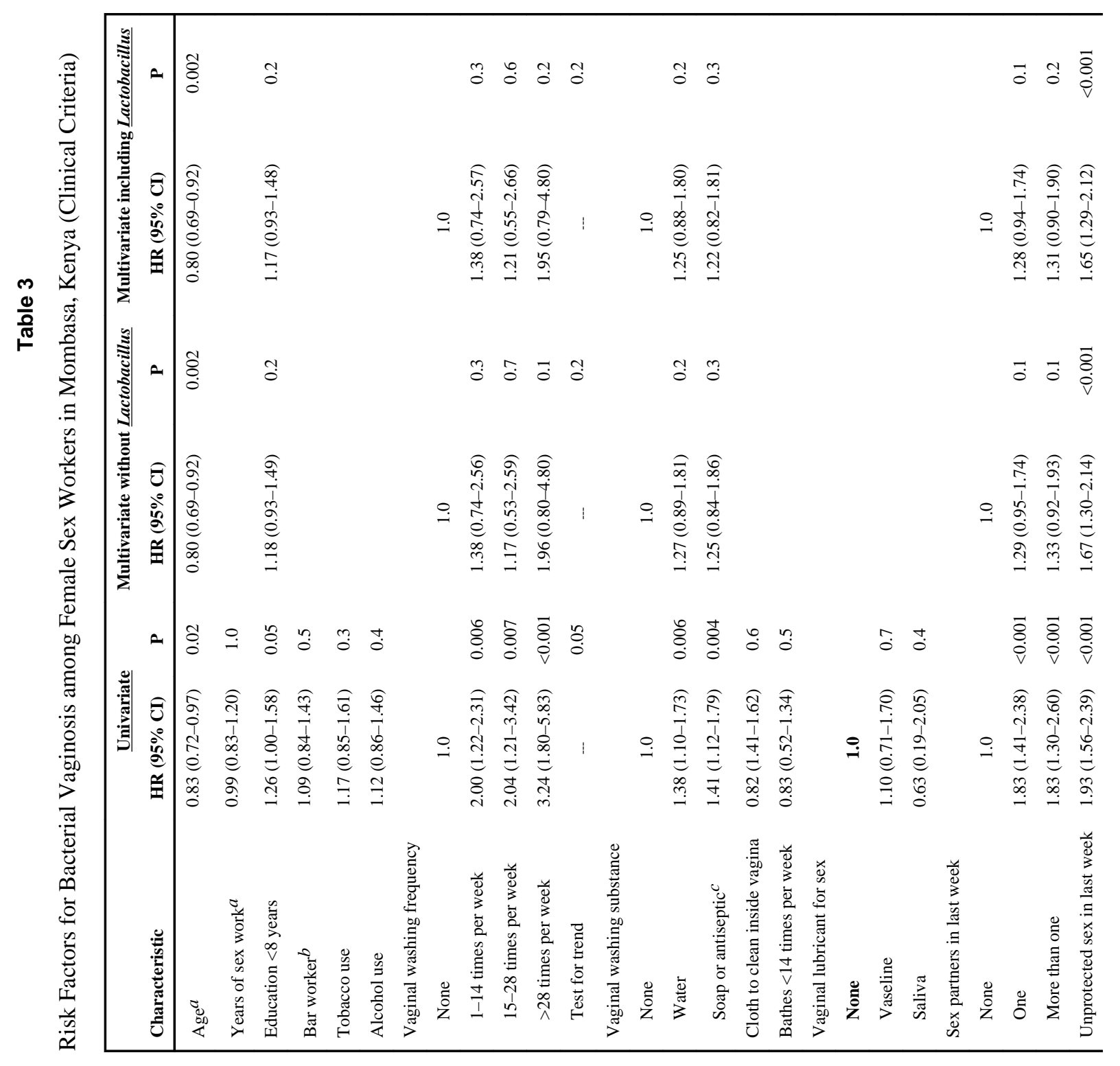




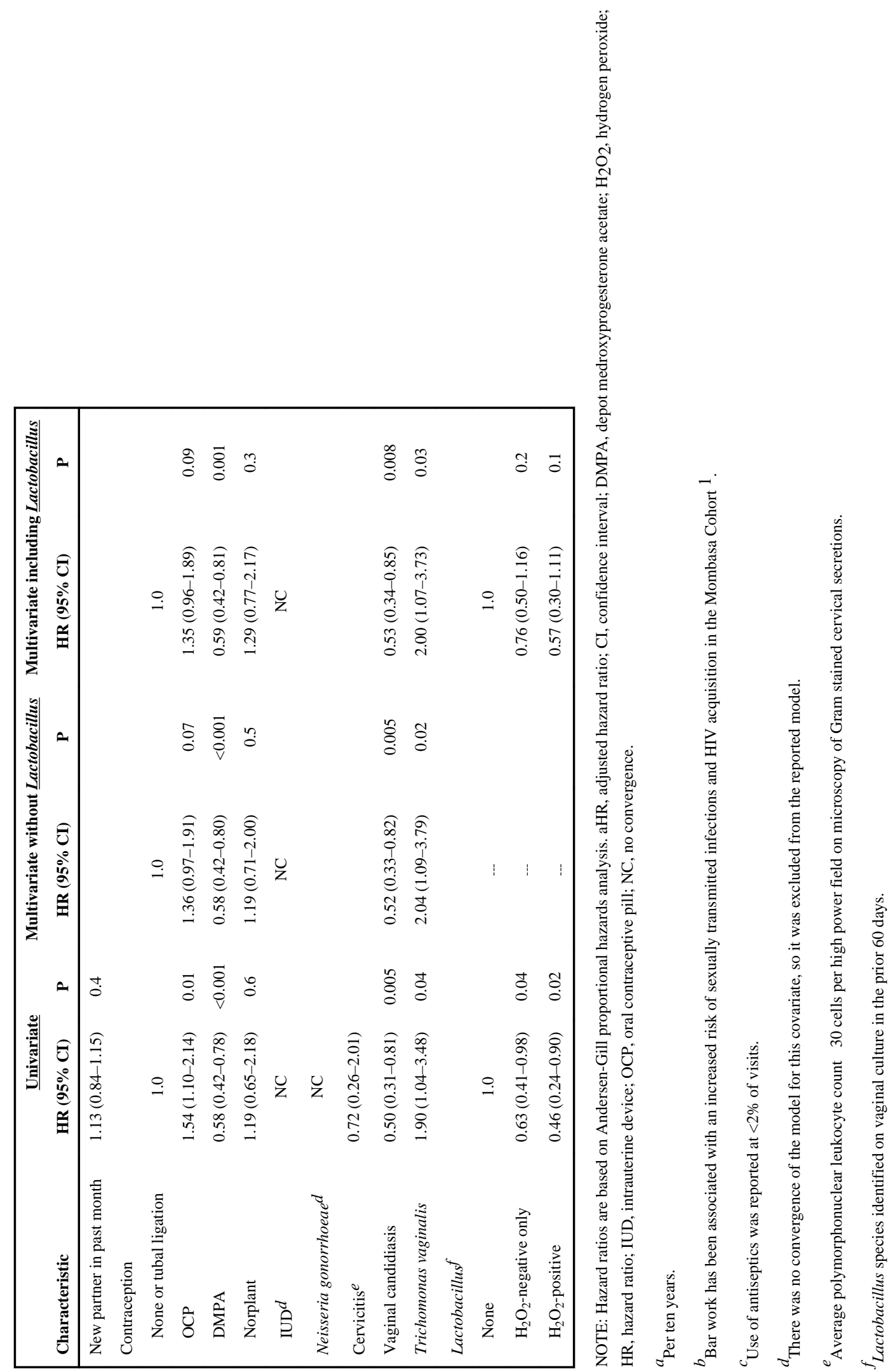

